Correspondence to D. S.

email:dsidrans@welchlink.welch.jhu.edu

Supported by grant CA 58-84-01 from the NCI and a collaborative research agreement with Oncor Inc, Gaithersburg, MD. J. J. is a recipient of The James Valvano Award for Cancer Research.

1. Hollstein, M, Sidransky, D., Vogelstein, B. \& Harris,
C. p53 mutations in human cancer. Science 253, 49-53 (1991).

2. Kaghad, M. et al. Monoalielically expressed gene related to $p 53$ at $1 \mathrm{p} 36$, a region frequently deleted in neuroblastoma and other human cancers. Cell 90 , 809-819 (1997).

3. Jost, A.C., Marin, C.M. \& Kaelin Ir., G.W. p73 is a human p53-related protein that can induce apoptosis. Nature 389, 191-194 (1997).

4. Schmale, H. \&Bamberger, C. A novel protein with strong homology to the tumor suppressor p53.

\section{Anti-KS activity still a mystery}

To the editor-We would like to comment on the description of an "hCG associated factor" (HAF) by Lunardi-Iskandar and colleagues in Nature Medicine (4, 428-434, 1998). This paper reports that the anti-HIV and anti-KS activities of crude hCG preparations co-purify in two peaks of a Superdex 200 column. One of these peaks closely corresponds to the 'beta-core' fragment of the hCG beta subunit. We have previously shown that beta-core from urine has an anti-KS activity (Albini et al. AIDS 11, 713-721, 1997). The beta-core preparation we used was purified by HPLC size exclusion, and thus very likely contained fractions equivalent to the larger 'HAF' fractions of Lunardi-Iskandar et al., since these peaks overlap almost completely.

Lunardi-Iskandar et al, noted that an apparently affinity purified beta-core, whose source was not given, was not active. They did not test the beta-core isolated from the HAF peak. We have found that the anti$\mathrm{KS}$ activity of the beta-core fraction is sensitive to storage conditions, being inactivated by repeated freeze-thaw cycles as well as by elution with concentrated acetic acid during affinity purification (Birken et al. Endocrinology 123, 572-583, 1988). The fact that Lunardi-Iskandar et al. found a single inactive beta-core preparation is not sufficient to demonstrate that the beta-core itself lacks anti-KS activity. The proposed lack of activity should have been ascertained by removal of the beta-core from the "HAF" fractions with antibodies recognizing the beta-core and testing for anti-KS activity. Given that this step is critical in identifying so-called HAF as a biological activity independent from the beta-core, we are very surprised that such relatively simple, yet very important, experiments were not done.

Although the authors imply that neither the beta-core nor the urinary RNase alone account for the anti-KS 'HAF' activity, they cannot exclude that the anti-KS activity is due to a cooperation between these two molecules, as originally suggested by Griffiths et al. (Nature 390, 598, 1997). The claims of Lunardi-Iskandar $e t$ al. that the beta-core and RNase have no anti-KS activity could only be demonstrated by physical separation of the anti-KS activity from these two factors, which was not done. The published characterization of 'HAF' is therefore much too preliminary to exclude that the anti-KS and anti-HIV activities within hCG are not, at least in part, due to the beta-core. Perhaps the authors should have pursued their original observation, and attempted to isolate their anti-KS activity from pregnant mice ( $\mathrm{O}^{\prime}$ Reilly et al., Cell 79, 315-328, 1994). Because mice do not make $h C G$, isolation of the murine factor would be definitive: in this case both Gallo's and our group would be wrong.

Douglas Noonan \& Adriana Albini

Istituto Nazionale per la Ricerca sul Cancro (IST) Centro di Biotecnologie Avanzate

Largo Rosanna Benzi, n.10, 16132 Genova Italy

Gallo et al. reply-We tested highly purified forms of hCG, subunits and hCG beta-core and found all to be completely inactive in all of our various assay systems. The hCG beta-core preparation that we tested was subjected to chemical sequencing and found to contain only the expected two chain hCG beta-core structure. It is true that the procedure we used exposed the betacore preparation to an acidic environment, however, the hCG beta core's immunoreactivity and structure survive acid treatment well as do the various ribonucleases. Both of these observations are incompatible with the observed acid lability of HAF. Noonan and Albini's suggested pass-through purification on immunoaffinity columns to evaluate which molecules may be involved in HAF activity would not be conclusive, because various other molecules (such as granulins, defensins, ribonucleases, lysozyme, etc.) also bind such columns.

We have also tested preparations of crude commercial hCG, which contain significant concentrations of the hCG beta-core but do not contain $\mathrm{HAF}$, another observation tending to exclude the involvement of the betacore. In addition, hCG beta-core is not
Oncogene 15, 1363-1367 (1997).

5. Li, $L$ \& et al. Identification of 3q21-22 deletions in urinary bladder carcinogenesis by superimposed histologic and genetic mapping. American Urologic Association Conference, An abstract (1998).

6. Bian, J.H. \& Sun, Y. p53CP, a putative $p 53$ competing protein that specifically binds to the consensus $p 53$ DNA binding sitesCa third member of the p53 family. Proc. Natl. Acad. Sci. USA 94, 14753-14758 (1997).

7. Zeiner, M. \& Gehrig, U. Cloning of $5^{\prime}$ CDNA regions by inverse PCR. Biotechniques 17, 1051-1053 (1994).

found in significant concentrations in blood, because it is a urinary product, yet $\mathrm{HAF}$ is found in sera from women in early pregnancy.

It is possible that the HAF activity may be due to a combination of molecules, a point we raised in the paper, but we do not have such information. Moreover, we know of no evidence for such effects. Indeed, we are surprised this interpretation was raised, since both the RNase and beta-core are available and it would be simple to test them together. We note again that HAF has three inseparable in vitro and in vivo activities (anti-KS, anti-HIV and pro-hematopoietic) and the activities are found in urine of early pregnancy. No evidence has been provided that RNase is present chiefly or solely in urine from early pregnancy or that it has a pro-hematopoietic effect, and no evidence has been provided that the hCG beta-core has anti-HIV or pro-hematopoietic effects. Rather than pursue possible candidate molecules for HAF activities, we think that it is more fruitful to isolate the molecules based on their bioactivities alone and then to identify their structures. Therefore, we will not make claims as to the identity of HAF until the structure of the purified molecules are known.

With respect to the suggestion that we attempt to isolate the anti-KS activity from pregnant mice, we disagree. We are interested in the factor(s) in humans which may not be the same as in mice. We have never claimed that the factor in mice and human are the same nor has anyone shown that the mouse factor has any anti-HIV or prohematopoietic effects. In fact we have not claimed to have identified the chemical nature of HAF. We have simply stated that it is not the beta-core of hCG, the hCG heterodimer itself or urinary RNase.

Robert C. Gallo ${ }^{1}$,YANTO LUNARDI-

ISKANDAR $^{1}$, JOSEPH BRYANT ${ }^{1}$

\& STEVEN BIRKEN ${ }^{2}$

${ }^{1}$ Institute of Human Virology,

University of Maryland, 725 W. Lombard Street,

Baltimore, MD, USA

${ }^{2}$ Columbia University

College of Physicians and Surgeons

630 West $168^{\text {th }}$ Street, New York, NY USA 